\title{
Associação entre qualidade do sono e o tempo de tela em adolescentes
}

\author{
Association between sleep quality and screen time in adolescents \\ Asociación entre la calidad del sueño y el tiempo frente a una pantalla en adolescentes
}

Recebido: 01/06/2021 | Revisado: 11/06/2021 | Aceito: 14/06/2021 | Publicado: 28/06/2021

\author{
Ademir Félix Arantes Júnior \\ ORCID: https://orcid.org/0000-0001-7986-1126 \\ Universidade de Pernambuco, Brasil \\ E-mail: ademirarantesjr@gmail.com \\ Sérgio Soares da Silva \\ ORCID: https://orcid.org/0000-0003-0303-6714 \\ Universidade de Pernambuco, Brasil \\ E-mail: sergio_oi007@yahoo.com.br \\ Emerllyn Shayane Martins de Araújo \\ ORCID: https://orcid.org/0000-0003-2763-7231 \\ Universidade de Pernambuco, Brasil \\ E-mail: emerllyn.shayane@upe.br \\ Mariana Araújo Coutinho da Silveira \\ ORCID: https://orcid.org/0000-0003-3365-5858 \\ Universidade de Pernambuco, Brasil \\ E-mail: marianaaraujocs@gmail.com \\ Mônica Vilela Heimer \\ ORCID: https://orcid.org/0000-0003-3842-192X \\ Universidade de Pernambuco, Brasil \\ E-mail: monica.vilelaheimer@gmail.com
}

\begin{abstract}
Resumo
Esta pesquisa teve como objetivo avaliar a associação entre a qualidade do sono e o tempo de tela em adolescentes escolares da cidade do Recife-PE. Realizou-se um estudo transversal com adolescentes entre 15 e 19 anos, do ensino Médio de uma escola da rede pública do Recife. Os dados foram coletados através de questionário digital utilizando a ferramenta Google Forms, empregando os seguintes questionários: Sociodemográfico; Índice de Qualidade de Sono de Pittsburgh (PSQI) e o Questionário de Mídias Eletrônicas. Foram utilizados os testes estatísticos Qui-quadrado de Pearson ou o teste Exato de Fisher, com intervalo de confiança de 95\% e um nível de significância de 5\%. A amostra foi composta por 128 adolescentes com idade média de 16,20 anos, sendo 62,5\% do sexo feminino. A maioria da amostra $(65,6 \%)$ apresentou uma duração do sono igual ou maior que 8 horas por noite e a qualidade do sono foi considerada boa por $62,5 \%$ dos pesquisados. O tempo de uso do celular $\geq 4$ horas/dia foi associado a má qualidade do sono. Houve associação da má qualidade do sono com o uso, após 10 horas da noite, de computadores (OR (IC à $95 \%) 10,6\left(2,6\right.$ a 42,0) $\left.\mathrm{p}^{(1)}<0,001^{*}\right)$, redes sociais (OR (IC à 95\%) 2,1 (2,4 a 16,1) $\mathrm{p}^{(1)}<0,001$ e televisão (OR (IC à $95 \%) 2,8(1,2$ a 6,2$) \mathrm{p}^{(1)}=0,011$. O uso noturno de telas digitais e um tempo de tela $\geq 4$ horas/dia foram associados a uma má qualidade do sono.
\end{abstract}

Palavras-chave: Qualidade do sono; Tempo de tela; Adolescente.

\begin{abstract}
In The aim of the present study was to evaluate the association between sleep quality and screen time among adolescent students. A cross-sectional study was conducted with adolescents between 15 and 19 years of age at a public high school in the city of Recife, Brazil. Data were collected through digital questionnaires using the Google Forms tool: questionnaire addressing sociodemographic characteristics, Pittsburgh Sleep Quality Index (PSQI) and Electronic Media Questionnaire. Statistical analysis involved either Pearson's chi-square test or Fisher's Exact test, with a 95\% confidence interval and 5\% significance level. The sample consisted of 128 adolescents (mean age: 16.20 years; $62.5 \%$ female). Most participants (65.6\%) slept eight or more hours per night and $62.5 \%$ considered their sleep quality to be good. Cellular phone use time $\geq 4$ hours/day was associated with poor sleep quality. Associations were also found between poor sleep quality and use after $10 \mathrm{pm}$ of computers [OR (95\% CI): 10.6 (2.6 to 42.0); $\mathrm{p}^{(1)}$ $<0.001$ ], social networks [OR (95\% CI): 2.1 (2.4 to 16.1); $\mathrm{p}^{(1)}<0.001$ ] and television [OR (95\% CI): 2.8 (1.2 to 6.2); $\left.\mathrm{p}^{(1)}=0.011\right]$. Nighttime use of digital screens and a screen time $\geq 4$ hours/day were associated with poor sleep quality.
\end{abstract}

Keywords: Sleep quality; Screen Time; Adolescent. 


\begin{abstract}
Resumen
Esta investigación tuvo como objetivo evaluar la asociación entre la calidad del sueño y el tiempo frente a la pantalla en adolescentes escolares de la ciudad de Recife-PE. Se realizó un estudio transversal con adolescentes de entre 15 y 19 años, de la escuela secundaria pública de Recife. Los datos fueron recolectados a través de un cuestionario digital utilizando la herramienta Google Forms, utilizando los siguientes cuestionarios: Sociodemográfico; Índice de calidad del sueño de Pittsburgh (PSQI) y el cuestionario de medios electrónicos. Se utilizó la prueba estadística Chi-cuadrado de Pearson o la prueba exacta de Fisher, con un intervalo de confianza del $95 \%$ y un nivel de significancia del $5 \%$. La muestra estuvo conformada por 128 adolescentes con una edad promedio de 16,20 años, de los cuales el 62,5\% eran mujeres. La mayoría de la muestra $(65,6 \%)$ tenía una duración del sueño igual o superior a 8 horas por noche y el $62,5 \%$ de los encuestados consideró buena la calidad del sueño. El tiempo de uso celular $\geq 4$ horas / día se asoció con una mala calidad del sueño. Hubo una asociación entre la mala calidad del sueño y el uso, después de las $10 \mathrm{pm}$, de computadoras (OR (IC del 95\%) 10,6 (2,6 a 42,0) $\mathrm{p}^{(1)}<0,001 *$ ), redes sociales (OR (IC del 95\%) 2,1 (2,4 a 16,1) $\mathrm{p}^{(1)}$ $<0,001$ y televisión (OR (IC del 95\%) 2,8 $\left(1,2\right.$ a 6,2) p ${ }^{(1)}=0,011$. El uso nocturno de pantallas digitales y un tiempo de pantalla $\geq 4$ horas / día se asociaron con una mala calidad del sueño.
\end{abstract}

Palabras clave: Calidad del sueño; Tiempo frente a la pantalla; Adolescente.

\title{
1. Introdução
}

Por ser um estado vital e complexo, o sono tem papel essencial na saúde da população, pois é caracterizado por processos ativos e organizados, que contribuem para restauração física e mental (Gajardo et al., 2021). Além disso, é fundamental no crescimento e na maturação durante a infância e adolescência, sendo essa última, a época em que o padrão do sono sofre mudanças fisiológicas consideráveis (Santos \& Souza,2021). Essa é uma fase da vida em que se observam grandes chances de desenvolver hábitos prejudiciais, como o uso demasiado de internet, por isso é importante estudar esse grupo etário e a associação com o uso progressivo dessa ferramenta (Schmidek et al., 2018).

Nas últimas décadas, a internet mudou a forma como os indivíduos se relacionam, adquirem conhecimento e trabalham. Embora diversos aspectos positivos e praticidades sejam disponibilizados para os usuários através dessa ferramenta, malefícios também podem ser vistos nos usuários que a utilizam em excesso (Schmidek et al., 2018). O uso demasiado da Internet vem sendo relacionado a consequências preocupantes e pode resultar em dependência (Zhang, Chen, Ye \& Volantin, 2020). Isso pode ocasionar problemas do sono em adolescentes e tais distúrbios são reconhecidos internacionalmente como uma grande preocupação de saúde (Otsuka et al., 2020). O Brasil encontra-se entre os cinco países com mais usuários ativos (Lobo, Gigante, Navarro, \& Raso, 2017).

A utilização excessiva de celulares como parte de mídias eletrônicas contribui para que os adolescentes não tenham uma higiene do sono adequada (Amra et al., 2017). Além disso, alguns itens como toques de mensagens recebidas durante a noite também acabam impossibilitando um sono de qualidade, visto que $86 \%$ dos adolescentes dormem com o telefone no quarto (Woods \& Scott, 2016).

Ressalta-se ainda que a luminosidade azul da tela dos dispositivos impede a produção de melatonina, modificando o ciclo circadiano, aumentando a excitação mental e fisiológica (Silva, Oliveira, Santos, \& Tassitano, 2017; Jniene et al., 2019). A melatonina, neuro-hormônio produzido pela glândula pineal, é responsável pela indução e manutenção do sono, e regula o ritmo circadiano, ciclo que possui a duração de 24 horas, período no qual se desempenham as atividades diárias e noturnas (Jniene et al., 2019; Calvo-Sanz \& Tapia-Ayuga, 2020; Glanzmann, Moreira, de Almeida Marques, Silva, \& Soares, 2019; Lira \& Custodio, 2018).

Evidências mostram que a demasiada exposição à tela causa menor tempo de duração do sono, afetando negativamente sua qualidade (Silva et al., 2017). De acordo com a literatura vigente, o ideal é que os adolescentes durmam de 8 a 10 horas de sono por noite (Kosticova, Husarova \& Dankulincova, 2020). No entanto, 30 a $50 \%$ dos adolescentes não dormem o suficiente e $10 \%$ e $20 \%$ apresentam a maior dificuldade na hora de adormecer ou permanecer dormindo (Kosticova et al., 2020). Diante do exposto, esse trabalho tem como objetivo avaliar a associação entre qualidade do sono e uso de telas digitais em adolescentes escolares da cidade do Recife-PE. 


\section{Metodologia}

Trata-se de um estudo observacional, com delineamento transversal. Este tipo de estudo envolve um recorte único do tempo e por isso não possuiu a capacidade de estabelecer relação de causa e efeito (Pereira, 1995).

A pesquisa foi realizada em uma escola, escolhida de modo intencional, da rede pública de ensino da cidade do Recife (PE). A cidade do Recife é capital do estado de Pernambuco. Possui uma população de 1.537.704 habitantes, destes 15,04\% encontram-se na faixa etária da adolescência segundo o Instituto Brasileiro de Geografia e Estatística [IBGE] (2010).

Participaram da pesquisa adolescentes de 15 a 19 anos, de ambos os sexos, regularmente matriculados do $1^{\circ}$ ao $3^{\circ}$ ano do Ensino Médio na rede pública do município do Recife (PE). Foram excluídos estudantes que estavam realizando algum tipo de tratamento para distúrbios do sono ou acompanhamento psicológico decorrente do transtorno de dependência de internet, ou que apresentaram limitações cognitivas que impossibilitaram a compreensão e aplicação dos instrumentos.

Em face da pandemia do COVID-19, e seguindo as orientações da Organização Mundial de Saúde - OMS, a coleta de dados foi realizada através de um questionário digital utilizando a ferramenta Google Forms, empregando os seguintes questionários: Sociodemográfico; Índice de Qualidade de Sono de Pittsburgh (PSQI); Questionário de Mídias Eletrônicas. O link do questionário digital, disponível para preenchimento por 15 dias, foi encaminhado para a diretora da instituição de ensino que, posteriormente, enviou para os estudantes através do aplicativo Whatsapp.

No questionário Sociodemográfico foram incluídas questões sobre idade (em anos), sexo, escolaridade, situação marital e situação profissional do responsável e renda familiar aproximada.

Foi utilizada a versão brasileira do Índice de Qualidade de Sono de Pittsburgh (PSQI), validado recentemente para o público adolescente com faixa etária de 10 a 19 anos de idade (Passos et al., 2017). Composto por 19 questões autorrelatadas, distribuídas em uma escala de 0 a 3, e organizada em sete componentes: (1) Qualidade subjetiva do sono; (2) latência do sono; (3) Duração do sono; (4) Eficiência habitual do sono; (5) Transtornos do sono; (6) Uso de medicamentos para dormir; (7) Disfunção diurna (Bertolazi et al., 2009). Os escores dos sete componentes devem ser somados para se obter a pontuação global, que varia de 0 a 21 pontos. Neste caso, quando o escore total for de 0 a 4, a qualidade do sono é boa; de 5 a 10 , a qualidade do sono é ruim; e > 10, significa que há presença de distúrbio do sono (Barbosa, Macedo, \& Silveira, 2011). Apresenta elevada consistência interna (Cronbach de 0,82) e confiabilidade moderada (Passos et al., 2017).

O Questionário de Mídias Eletrônicas é um questionário individual, confidencial e autoaplicável, com perguntas acerca do uso de telas digitais e separadas em blocos categorizáveis. As telas digitais avaliadas são os computadores, celulares e videogames, além da TV, abordando os aspectos de disponibilidade e padrão de consumo. O questionário é validado e foi utilizado também como pré-teste em estudo anterior (Queiroz, Lourenço, Silva, Lourenço, \& Silva, 2018), no qual foi adaptado para garantir o entendimento dos alunos e avaliar sua aplicabilidade. O questionário tem duração média de 30 minutos para respostas. Para a obtenção dos resultados foi considerado o tempo de tela escritos pelos entrevistados em horas, e a avaliação do uso de mídias digitais e sua frequência. Com os blocos separados e categorizáveis, a avaliação dos dados coletados foi descrita em porcentagem.

Para a análise dos dados foram utilizados os testes Qui quadrado de Pearson e Exato de Fisher. Para avaliar a força da associação foram obtidos o OR (Odds Ratio) ou RC (Razão das Chances) nas tabelas bivariadas e seus respectivos intervalos de confiança. O nível de significância utilizada na decisão dos testes estatísticos foi de 5\% e os intervalos foram obtidos com 95\% de confiança. Os dados foram digitados na planilha EXCEL e o programa utilizado para obtenção dos cálculos estatísticos foi o IMB SPSS na versão 25 .

Este trabalho foi aprovado pelo Comitê de Ética em Pesquisa da Universidade de Pernambuco - UPE, sob o parecer 3. 242.121. Solicitou-se que cada participante assinalasse o item referente à concordância do Termo de Consentimento Livre e Esclarecido (TCLE), para os adolescentes com idade maior ou igual a 18 anos. Para os menores, além do TCLE respondido 
pelos responsáveis, era necessário a concordância do menor através do Termo de Assentimento Livre e Esclarecido (TALE). Assim, os sujeitos só puderam participar da pesquisa após a marcação relativa à concordância dos termos.

Os riscos poderiam vir a ocorrer caso o aluno ou responsável sentisse constrangido em responder os questionários. No entanto, os questionários foram aplicados por meio de um link do Google Forms, enviado através do aplicativo WhatsApp, e respondidos sob sigilo, sem a presença de professores (as) e/ou diretores e pesquisadores. Os benefícios estão relacionados à aquisição de informações sobre a qualidade do sono e o tempo de tela dos adolescentes, trazendo mais conhecimento sobre esta temática, podendo contribuir para ações de políticas de prevenção e promoção de saúde para este público.

\section{Resultados}

Os adolescentes que responderam o formulário de forma incompleta foram considerados como perda. Dessa forma, a amostra final foi composta por 128 adolescentes.

Na Tabela 1 observa-se os resultados relativos às características dos pesquisados, onde pode ser verificado que a maioria dos adolescentes $(63,3 \%)$ tinha 15 a 16 anos de idade, estava no $1^{\circ}$ ano do ensino médio (55,5), era do sexo feminino $(62,5 \%)$ e todos estudavam no turno da manhã. Em relação aos responsáveis, o maior percentual foi de solteiro (46,9\%), estava trabalhando $(52,3 \%)$ e tinha renda familiar de 1 a 3 salários mínimos $(64,8 \%)$.

Tabela 1 - Característica da amostra analisada.

\begin{tabular}{|c|c|c|}
\hline Variável & $N$ & $\%$ \\
\hline Total & 128 & 100,0 \\
\hline \multicolumn{3}{|l|}{ Idade } \\
\hline 15 a 16 & 81 & 63,3 \\
\hline 17 a 19 & 47 & 36,7 \\
\hline \multicolumn{3}{|c|}{ Ano (Ensino médio) } \\
\hline $1^{\circ}$ & 71 & 55,5 \\
\hline $2^{\circ}$ & 20 & 15,6 \\
\hline $3^{\circ}$ & 37 & 28,9 \\
\hline \multicolumn{3}{|l|}{ Turno } \\
\hline Manhã & 128 & 100,0 \\
\hline \multicolumn{3}{|l|}{ Sexo } \\
\hline Masculino & 48 & 37,5 \\
\hline Feminino & 80 & 62,5 \\
\hline \multicolumn{3}{|c|}{ Situação marital do responsável } \\
\hline Solteiro & 60 & 46,9 \\
\hline Casado & 50 & 39,1 \\
\hline Separado/ viúvo & 18 & 14,0 \\
\hline \multicolumn{3}{|c|}{ Situação profissional do responsável } \\
\hline Desempregado & 61 & 47,7 \\
\hline Trabalhando & 67 & 52,3 \\
\hline \multicolumn{3}{|c|}{ Renda familiar (salários mínimos) } \\
\hline Menos de 1 & 40 & 31,3 \\
\hline 1 a 3 & 83 & 64,8 \\
\hline Mais de 3 & 5 & 3,9 \\
\hline
\end{tabular}

Fonte: Autores. 
Observou-se que a maioria apresentou 8 ou mais horas de sono por noite $(65,6 \%)$ e não referiu dor $(48,4 \%)$. Em relação a qualidade do sono, $37,5 \%$ consideraram ruim e $78,1 \%$ do grupo afirmou ter dificuldade em manter o entusiasmo (ânimo) para fazer as atividades habituais (Tabela 2)

Questionados se dividiam o quarto com alguém, 35,9\% responderam afirmativamente e neste subgrupo, segundo informações da pessoa que dormia no mesmo quarto, 23,9\% afirmaram que tinham ronco forte, 21,7\% tinham longas paradas na respiração, 41,3\% apresentaram contrações ou puxões nas pernas e 34,8\% reportaram ter episódios de desorientação ou confusão durante o sono (Tabela 2).

Tabela 2 - Avaliação da qualidade do sono.

\begin{tabular}{|c|c|c|}
\hline Variável & $N$ & $\%$ \\
\hline \multicolumn{3}{|c|}{ Horas de sono por noite (Variável gerada a partir da última variável) } \\
\hline Menos de 8horas & 44 & 34,4 \\
\hline 8 horas ou mais & 84 & 65,6 \\
\hline \multicolumn{3}{|l|}{ Teve dor } \\
\hline Nenhuma vez no último mês & 62 & 48,4 \\
\hline Menos de uma vez por semana & 24 & 18,8 \\
\hline Uma ou duas vezes por semana & 27 & 21,1 \\
\hline Três ou mais vezes por semana & 15 & 11,7 \\
\hline \multicolumn{3}{|c|}{ Durante o último mês, como classificaria a qualidade do seu sono de uma maneira geral? } \\
\hline Boa & 80 & 62,5 \\
\hline Ruim & 48 & 37,5 \\
\hline \multicolumn{3}{|c|}{$\begin{array}{l}\text { Dificuldade em manter o entusiasmo (ânimo) para fazer as atividades habituais (No } \\
\text { último mês) }\end{array}$} \\
\hline Sim & 100 & 78,1 \\
\hline Não & 28 & 21,9 \\
\hline \multicolumn{3}{|c|}{ Tem um(a) parceiro(a) (cônjuge) ou colega de quarto? } \\
\hline Sim & 46 & 35,9 \\
\hline Não & 82 & 64,1 \\
\hline TOTAL & 128 & 100,0 \\
\hline \multicolumn{3}{|c|}{ Teve um ronco forte segundo informações do parceiro ou colega? ${ }^{(1)}$} \\
\hline Sim & 11 & 23,9 \\
\hline Não & 35 & 76,1 \\
\hline \multicolumn{3}{|c|}{ Teve longas paradas enquanto dormia? ${ }^{(1)}$} \\
\hline Sim & 10 & 21,7 \\
\hline Não & 36 & 78,3 \\
\hline \multicolumn{3}{|c|}{ Teve contrações ou puxões nas pernas enquanto dormia? ${ }^{(1)}$} \\
\hline Sim & 19 & 41,3 \\
\hline Não & 27 & 58,7 \\
\hline \multicolumn{3}{|c|}{ Teve episódios de desorientação ou confusão enquanto dormia? ${ }^{(1)}$} \\
\hline Sim & 16 & 34,8 \\
\hline Não & 30 & 65,2 \\
\hline TOTAL & 46 & 100,0 \\
\hline
\end{tabular}

(1) os valores percentuais foram obtidos com base no total de 46 participantes que dormem em companhia de uma outra pessoa. Fonte: Autores. 
Na Tabela 3 é possível verificar que 49,2\% utilizavam computador. Dentre esses, 44,4\% utilizavam todos os dias da semana, 39,7\% afirmaram utilizar 4 horas ou mais por dia, de segunda a sexta feira e este percentual aumentou para $76,2 \%$ aos sábados, domingos e feriados. Questionados se costumavam utilizar o computador após as 10 horas da noite, quase a metade $(49,2 \%)$ respondeu afirmativamente.

Tabela 3 - Avaliação da frequência do uso do computador.

\begin{tabular}{lll} 
Variável & $N \quad \% \quad \%$ \\
\hline
\end{tabular}

Tipo de computador que mais usa?

Computador portátil (notebook ou laptop)

Computador de mesa (desktop)

TOTAL

$\begin{array}{cc}65 & 50,8 \\ 52 & 40,6 \\ 11 & 8,6 \\ \mathbf{1 2 8} & \mathbf{1 0 0 , 0}\end{array}$

Quantos dias por semana, usa computador?

Um dia

2 a 6

$13 \quad 20,6$

Todos os dias

De segunda à sexta-feira, quanto tempo, em média, você fica no computador?

Menos de $4 \mathrm{~h}$

4h ou mais

$25 \quad 39,7$

Aos sábados, domingos e feriados, quanto tempo, em média, você fica no computador?

Menos de 4h/dia $\quad 15 \quad 23,8$

\begin{tabular}{ll} 
4h/dia ou mais & 48 \\
\hline
\end{tabular}

$\begin{array}{lrr}\text { Costuma usar o computador após } 10 \text { horas da noite? } & 31 & 49,2 \\ \text { Sim } & 32 & 50,8 \\ \text { Não } & \mathbf{6 3} & \mathbf{1 0 0 , 0} \\ \text { TOTAL } & \end{array}$

Fonte: Autores.

A Tabela 4 apresenta os dados referentes ao uso de jogos eletrônicos. Nesta tabela é possível verificar que a maioria dos adolescentes jogavam (70,3\%) e dentre esses, 51,1\% jogavam 1 a 3 dias por semana. O percentual dos que jogavam em média 4 horas ou mais por dia, de segunda a sexta-feira foi 32,2\%, passando para 43,3\% aos sábados, domingos e feriados. A maioria $(56,7 \%)$ costumava jogar após as 10 horas da noite. 
Tabela 4 - Avaliação da frequência do uso dos jogos eletrônicos.

\begin{tabular}{|c|c|}
\hline Variável & $N$ \\
\hline
\end{tabular}

\section{Joga jogos eletrônicos?}

Sim

Não

Quantos dias na semana habitualmente você joga jogos eletrônicos?

1 a 3

4 a 6

Todos os dias

De segunda a sexta-feira, quanto tempo, em média, você joga jogos eletrônicos?

Menos de 4h/dia

$4 \mathrm{~h} /$ dia ou mais

Aos sábados, domingos e feriados, quanto tempo, em média, você joga jogos eletrônicos?

Menos de 4h/dia $\quad 51 \quad 56,7$

4h/dia ou mais $\quad 39 \quad 33,3$

$\begin{array}{lrr}\text { Costuma jogar jogos eletrônicos após às } \mathbf{1 0} \text { horas da noite? } & 51 & 56,7 \\ \text { Sim } & 39 & 43,3 \\ \text { Não } & \mathbf{9 0} & \mathbf{1 0 0 , 0} \\ \text { TOTAL } & \end{array}$

Fonte: Autores.

Sobre o acesso às redes sociais, observa-se que todos responderam participar de alguma rede social, 36,7\% começaram a participar entre 10 e 12 anos de idade, 96,1\% citaram o celular como o equipamento que mais utilizava para acessar a rede social e 81,2\% costumavam se conectar após às 10 horas da noite. Em relação as funções realizadas com o celular, 35,9\% e 34,4 referiu, respectivamente, acesso à internet e ao WhatsApp. A maioria (83,6\%) utilizava 4 ou mais horas por dia, de segunda a sexta, passando para 85,9\% aos sábados, domingos e feriados. Quase 90\% dos adolescentes costumavam utilizar o celular após as 10 horas da noite. De segunda a sexta 27,3\% costumavam assistir TV mais de 4 horas por dia, passando para 31,3\% aos sábados, domingos e feriados. Exatamente a metade dos pesquisados afirmou assistir TV após as 10 horas da noite. Questionados sobre quais dispositivos que frequentemente usam ao mesmo tempo, 40,6\% responderam celular e TV (Tabela 5). 
Tabela 5 - Avaliação da frequência ao acesso às redes sociais.

\begin{tabular}{|c|c|c|}
\hline Variável & $N$ & $\%$ \\
\hline TOTAL & 128 & 100,0 \\
\hline Participa de alguma rede social? & 128 & 100,0 \\
\hline \multicolumn{3}{|l|}{ Com que idade começou a participar de rede social? } \\
\hline Não lembra & 34 & 26,6 \\
\hline Menos de 10 anos & 12 & 9,4 \\
\hline 10 a 12 anos & 47 & 36,7 \\
\hline 13 a 15 anos & 33 & 25,8 \\
\hline 16 a 19 anos & 2 & 1,5 \\
\hline \multicolumn{3}{|c|}{ Que equipamento mais utiliza para acessar a rede social? } \\
\hline Telefone celular com acesso à internet & 123 & 96,1 \\
\hline Computador portátil (Notebook, Laptop ou Tablet) & 4 & 3,1 \\
\hline Computador de mesa & 1 & 0,8 \\
\hline \multicolumn{3}{|c|}{ Costuma se conectar a uma rede social após às 10 horas da noite? } \\
\hline Sim & 104 & 81,2 \\
\hline Não & 24 & 18,8 \\
\hline \multicolumn{3}{|c|}{ Além de fazer e/ou receber chamadas, para que você mais usa o celular? } \\
\hline Acessar a internet & 46 & 35,9 \\
\hline WhatsApp & 44 & 34,4 \\
\hline Assistir vídeos & 15 & 11,7 \\
\hline Jogar & 12 & 9,4 \\
\hline Ouvir músicas & 10 & 7,8 \\
\hline Ler & 1 & 0,8 \\
\hline \multicolumn{3}{|c|}{ De segunda à sexta-feira, quanto tempo, em média, usa celular? } \\
\hline Menos de $4 \mathrm{~h} /$ dia & 21 & 16,4 \\
\hline 4h/dia ou mais & 107 & 83,6 \\
\hline \multicolumn{3}{|c|}{ Aos sábados, domingos e feriados, quanto tempo, em média, usa o celular? } \\
\hline Menos de 4h/dia & 18 & 14,1 \\
\hline $4 \mathrm{~h} / \mathrm{dia}$ ou mais & 110 & 85,9 \\
\hline \multicolumn{3}{|l|}{ Costuma usar o celular após às 10 horas da noite? } \\
\hline Sim & 115 & 89,8 \\
\hline Não & 13 & 10,2 \\
\hline \multicolumn{3}{|c|}{ De segunda à sexta-feira, quanto tempo, em média, costuma assistir TV? } \\
\hline Menos de $4 \mathrm{~h} / \mathrm{dia}$ & 93 & 72,7 \\
\hline $4 \mathrm{~h} / \mathrm{dia}$ ou mais & 35 & 27,3 \\
\hline \multicolumn{3}{|c|}{ Aos sábados, domingos e feriados, quanto tempo, em média, costuma assistir TV? } \\
\hline Menos de 4h/dia & 88 & 68,7 \\
\hline 4h/dia ou mais & 40 & 31,3 \\
\hline \multicolumn{3}{|l|}{ Costuma assistir TV após às 10 horas da noite? } \\
\hline Sim & 64 & 50,0 \\
\hline Não & 64 & 50,0 \\
\hline \multicolumn{3}{|c|}{ Assinale abaixo quais dispositivos, que frequentemente usa ao mesmo tempo? } \\
\hline Celular & 47 & 36,7 \\
\hline Celular + TV & 52 & 40,6 \\
\hline Notebook ou laptop + Celular & 11 & 8,6 \\
\hline Notebook ou laptop + Celular $+\mathrm{TV}$ & 8 & 6,3 \\
\hline Computador de mesa + Celular & 4 & 3,1 \\
\hline Notebook ou laptop + TV & 2 & 1,6 \\
\hline Notebook ou laptop + Tablet + Celular & 1 & 0,8 \\
\hline Notebook ou laptop + Tablet + Celular + TV & 1 & 0,8 \\
\hline Tablet + Celular & 1 & 0,8 \\
\hline Computador de mesa + Celular $+\mathrm{TV}$ & 1 & 0,8 \\
\hline
\end{tabular}

Fonte: Autores. 
Na Tabela 6 verifica-se que a má qualidade do sono foi associada ao uso de computador após às 10 horas da noite (p < 0,001).

Tabela 6 - Avaliação da qualidade do sono, segundo o uso do computador e se costuma jogar jogos eletrônicos.

\begin{tabular}{|c|c|c|c|c|c|c|c|c|}
\hline \multirow{3}{*}{ Variável } & \multicolumn{7}{|c|}{ Qualidade do sono } & \multirow{3}{*}{ Valor de p } \\
\hline & \multicolumn{2}{|c|}{ Má } & \multicolumn{2}{|c|}{ Boa } & \multicolumn{2}{|c|}{ Total } & OR (IC à & \\
\hline & $\mathrm{n}$ & $\%$ & $\mathrm{~N}$ & $\%$ & $\mathrm{n}$ & $\%$ & & \\
\hline Tipo de computador que mais usa? & & & & & & & & $\mathrm{p}^{(1)}=0,735$ \\
\hline Não usa & 48 & 73,8 & 17 & 26,2 & 65 & 100,0 & $1,1(0,3$ a 4,5$)$ & \\
\hline $\begin{array}{l}\text { Computador portátil (notebook ou } \\
\text { laptop) }\end{array}$ & 35 & 67,3 & 17 & 32,7 & 52 & 100,0 & $0,8(0,2$ a 3,3$)$ & \\
\hline Computador de mesa (desktop) & 8 & 72,7 & 3 & 27,3 & 11 & 100,0 & 1,0 & \\
\hline Grupo total & 91 & 71,1 & 37 & 28,9 & 128 & 100,0 & & \\
\hline $\begin{array}{l}\text { Costuma usar o computador após } 10 \\
\text { horas da noite? }\end{array}$ & & & & & & & & $\begin{array}{c}\mathrm{p}^{(1)}< \\
0,001 *\end{array}$ \\
\hline Sim & 28 & 90,3 & 3 & 9,7 & 31 & 100,0 & $\begin{array}{c}10,6(2,6 \mathrm{a} \\
42,0)\end{array}$ & \\
\hline Não & 15 & 46,9 & 17 & 53,1 & 32 & 100,0 & 1,0 & \\
\hline Grupo total & 43 & 68,3 & 20 & 31,7 & 63 & 100,0 & & \\
\hline Joga jogos eletrônicos? & & & & & & & & $\mathrm{p}^{(1)}=0,390$ \\
\hline Sim & 66 & 73,3 & 24 & 26,7 & 90 & 100,0 & $1,4^{\prime}(0,6$ a 3,2$)$ & \\
\hline Não & 25 & 65,8 & 13 & 34,2 & 38 & 100,0 & 1,0 & \\
\hline Grupo total & 91 & 71,1 & 37 & 28,9 & 128 & 100,0 & & \\
\hline $\begin{array}{l}\text { Costuma jogar jogos eletrônicos após } \\
10 \text { horas da noite? }\end{array}$ & & & & & & & & $\mathrm{p}^{(1)}=0,211$ \\
\hline Sim & 40 & 78,4 & 11 & 21,6 & 51 & 100,0 & $1,8(0,7$ a 4,7$)$ & \\
\hline Não & 26 & 66,7 & 13 & 33,3 & 39 & 100,0 & $+1,0$ & \\
\hline Grupo total & 66 & 73,3 & 24 & 26,7 & 90 & 100,0 & & \\
\hline
\end{tabular}

(*) Associação significativa ao nível de 5,0\%

(1) Pelo teste Qui-quadrado de Pearson.

Fonte: Autores.

Na Tabela 7 se verifica que os adolescentes que se conectavam a uma rede social após as 10 horas da noite tinham duas vezes mais chances de apresentar uma má qualidade do sono $\{2,1(2,4$ a 16,1$)\} \mathrm{p}^{(1)}<0,001$. 
Tabela 7 - Avaliação da qualidade do sono, segundo a frequência ao acesso às redes sociais.

\begin{tabular}{|c|c|c|c|c|c|c|c|c|}
\hline \multirow{3}{*}{ Variável } & \multicolumn{6}{|c|}{ Qualidade do sono } & \multirow{3}{*}{$\begin{array}{c}\text { OR (IC à } \\
95 \%)\end{array}$} & \multirow{3}{*}{ Valor de $p$} \\
\hline & \multicolumn{2}{|c|}{ Má } & \multicolumn{2}{|c|}{ Boa } & \multicolumn{2}{|c|}{ Total } & & \\
\hline & $\mathrm{n}$ & $\%$ & $\mathrm{~N}$ & $\%$ & $\mathrm{n}$ & $\%$ & & \\
\hline Grupo total & 91 & 71,1 & 37 & 28,9 & 128 & 100,0 & & \\
\hline $\begin{array}{l}\text { Com que idade começou a } \\
\text { participar de rede social? }\end{array}$ & & & & & & & & $\mathrm{p}^{(1)}=0,089$ \\
\hline Não lembra & 23 & 67,6 & 11 & 32,4 & 34 & 100,0 & $1,1(0,4$ a 3,0$)$ & \\
\hline Menos de 10 anos & 6 & 50,0 & 6 & 50,0 & 12 & 100,0 & $0,5(0,1$ a 2,0$)$ & \\
\hline 10 a 12 anos & 39 & 83,0 & 8 & 17,0 & 47 & 100,0 & $2,5(0,9$ a 7,1$)$ & \\
\hline 13 ou mais & 23 & 65,7 & 12 & 34,3 & 35 & 100,0 & 1,0 & \\
\hline $\begin{array}{l}\text { Costuma se conectar a uma rede } \\
\text { social após às } 10 \text { horas da noite? }\end{array}$ & & & & & & & & $\mathrm{p}^{(1)}<0,001^{*}$ \\
\hline $\operatorname{Sim}$ & 82 & 78,8 & 22 & 21,2 & 104 & 100,0 & $\begin{array}{c}2,1(2,4 \mathrm{a} \\
16,1)\end{array}$ & \\
\hline Não & 9 & 37,5 & 15 & 62,5 & 24 & 100,0 & 1,0 & \\
\hline $\begin{array}{l}\text { Além de fazer e/ou receber } \\
\text { chamadas, para que você mais } \\
\text { usa o celular? }\end{array}$ & & & & & & & & $\mathrm{p}^{(2)}=0,059$ \\
\hline Acessar a internet & 35 & 76,1 & 11 & 23,9 & 46 & 100,0 & $* *$ & \\
\hline WhatsApp & 33 & 75,0 & 11 & 25,0 & 44 & 100,0 & $* *$ & \\
\hline Assistir vídeos & 10 & 66,7 & 5 & 33,3 & 15 & 100,0 & $* *$ & \\
\hline Jogar & 4 & 33,3 & 8 & 66,7 & 12 & 100,0 & $* *$ & \\
\hline Ouvir músicas/Ler & 9 & 81,8 & 2 & 18,2 & 11 & 100,0 & $* *$ & \\
\hline
\end{tabular}

(*) Associação significativa ao nível de 5,0\%

(**) Não possível determinar devido à ocorrência de frequência muito baixa

(1) Pelo teste Qui-quadrado de Pearson

(2) Pelo teste Exato de Fisher.

Fonte: Autores

Observou-se que os adolescentes que ficavam no celular 4 horas ou mais, durante a semana ou nos fins de semana, apresentavam má qualidade do sono (respectivamente, $\mathrm{p}=0,002$ e p=0,007). Aqueles que ficavam no celular após as 10:00 horas da noite tiveram sete vezes mais chances de apresentar má qualidade do sono. Da mesma forma, os adolescentes que costumavam assistir TV após às 10:00 horas da noite tiveram quase três vezes mais chances de ter uma má qualidade do sono (Tabela 8) 
Tabela 8 - Avaliação da qualidade do sono, segundo a frequência do uso de aparelho eletrônicos.

\begin{tabular}{|c|c|c|c|c|c|c|c|c|}
\hline \multirow{3}{*}{ Variável } & \multicolumn{6}{|c|}{ Qualidade do sono } & \multirow{3}{*}{ OR (IC à 95\%) } & \multirow{3}{*}{ Valor de $p$} \\
\hline & \multicolumn{2}{|c|}{ Má } & \multicolumn{2}{|c|}{ Boa } & \multicolumn{2}{|c|}{ Total } & & \\
\hline & $\mathrm{n}$ & $\%$ & $\mathrm{~N}$ & $\%$ & $\mathrm{~N}$ & $\%$ & & \\
\hline Grupo total & 91 & 71,1 & 37 & 28,9 & 128 & 100,0 & & \\
\hline $\begin{array}{l}\text { De segunda à sexta-feira, quanto } \\
\text { tempo, em média, usa celular? }\end{array}$ & & & & & & & & $\begin{array}{l}\mathrm{p}^{(1)}= \\
0,002 *\end{array}$ \\
\hline Menos de 4h/dia & 9 & 42,9 & 12 & 57,1 & 21 & 100,0 & 1,0 & \\
\hline $4 \mathrm{~h} /$ dia ou mais & 82 & 76,6 & 25 & 23,4 & 107 & 100,0 & $4,4(1,7$ a 11,6$)$ & \\
\hline $\begin{array}{l}\text { Aos sábados, domingos e feriados, } \\
\text { quanto tempo, em média, usa o } \\
\text { celular? }\end{array}$ & & & & & & & & $\begin{array}{c}\mathrm{p}^{(1)}= \\
0,007 *\end{array}$ \\
\hline Menos de $4 \mathrm{~h} / \mathrm{dia}$ & 8 & 44,4 & 10 & 55,6 & 18 & 100,0 & 1,0 & \\
\hline 4h/dia ou mais & 83 & 75,5 & 27 & 24,5 & 110 & 100,0 & $3,8(1,4$ a 10,7$)$ & \\
\hline $\begin{array}{l}\text { Costuma usar o celular após às } 10 \\
\text { horas da noite? }\end{array}$ & & & & & & & & $\begin{array}{c}\mathrm{p}^{(2)}= \\
0,002 *\end{array}$ \\
\hline Sim & 87 & 75,7 & 28 & 24,3 & 115 & 100,0 & $7,0(2,0$ a 24,5$)$ & \\
\hline Não & 4 & 30,8 & 9 & 69,2 & 13 & 100,0 & 1,0 & \\
\hline $\begin{array}{l}\text { De segunda à sexta-feira, quanto } \\
\text { tempo, em média, costuma } \\
\text { assistir TV? }\end{array}$ & & & & & & & & $\mathrm{p}^{(1)}=0,354$ \\
\hline Menos de 4h/dia & 64 & 68,8 & 29 & 31,2 & 93 & 100,0 & 1,0 & \\
\hline 4h/dia ou mais & 27 & 77,1 & 8 & 22,9 & 35 & 100,0 & $1,5(0,6$ a 3,8$)$ & \\
\hline $\begin{array}{l}\text { Aos sábados, domingos e feriados, } \\
\text { quanto tempo, em média, costuma } \\
\text { assistir TV? }\end{array}$ & & & & & & & & $\mathrm{p}^{(1)}=0,281$ \\
\hline Menos de $4 \mathrm{~h} / \mathrm{dia}$ & 60 & 68,2 & 28 & 31,8 & 88 & 100,0 & 1,0 & \\
\hline 4h/dia ou mais & 31 & 77,5 & 9 & 22,5 & 40 & 100,0 & $1,6(0,7$ a 3,8$)$ & \\
\hline $\begin{array}{l}\text { Costuma assistir TV após às } 10 \\
\text { horas da noite? }\end{array}$ & & & & & & & & $\begin{array}{c}\mathrm{p}^{(1)}= \\
0,011^{*}\end{array}$ \\
\hline Sim & 52 & 81,2 & 12 & 18,8 & 64 & 100,0 & $2,8(1,2$ a 6,2$)$ & \\
\hline Não & 39 & 60,9 & 25 & 39,1 & 64 & 100,0 & 1,0 & \\
\hline $\begin{array}{l}\text { Assinale abaixo quais } \\
\text { dispositivos, que frequentemente } \\
\text { usa ao mesmo tempo? }\end{array}$ & & & & & & & & $\mathrm{p}^{(1)}=0,070$ \\
\hline Celular & 28 & 59,6 & 19 & 40,4 & 47 & 100,0 & 1,0 & \\
\hline Celular + TV & 42 & 80,8 & 10 & 19,2 & 52 & 100,0 & $2,9(1,2$ a 7,0$)$ & \\
\hline Outras combinações & 21 & 72,4 & 8 & 27,6 & 29 & 100,0 & $1,8(0,7$ a 4,8$)$ & \\
\hline
\end{tabular}

\footnotetext{
(*) Associação significativa ao nível de 5,0\%

(1) Pelo teste Qui-quadrado de Pearson

(2) Pelo teste Exato de Fisher.

Fonte: Autores.
}

\section{Discussão}

Os achados da presente pesquisa demonstraram que a maioria dos estudantes referiu ter 8 ou mais horas de sono por noite, o que corrobora com American Academy of Sleep Medicine [AASM] (2016) que preconiza que adolescentes de 13 a 18 anos tenham de 8 a 10 horas de sono por noite, para ter uma melhor qualidade de vida (Kosticova et al., 2020; Paruthi et al., 2016). No entanto, apesar da quantidade adequada de horas de sono, boa parte dos pesquisados apresentou má qualidade do sono, indicando que outros fatores podem levar a um sono de baixa qualidade, como por exemplo, a ingestão de bebidas energéticas, de cafeína, o sedentarismo e o alto índice de gordura corporal (Barbosa et al., 2020). 
O tempo prolongado de uso do celular para acesso à internet foi associado à má qualidade do sono, principalmente após as 10 horas da noite. Resultados semelhantes foram relatados por alguns autores (Mesquita \& Reimão, 2007; Munezawa et al., 2011; Polos et al., 2015; Lin et al., 2019) que verificaram que o uso de telefones celulares após apagar das luzes, ou durante os horários normais de sono, está associado ao sono de curta duração, sonolência diurna excessiva e insônia que, por sua vez, estão associados à má qualidade do sono.

No presente estudo foi observado que 26,6\% dos adolescentes não lembraram quando começaram a utilizar as redes sociais, cujo dado sugere que a inserção nessas redes tem acontecido precocemente. Essa informação é explicada por Neves, Fosse, Torres e Napolitano (2015), que afirmam que as mídias sociais fazem parte da realidade da sociedade contemporânea, principalmente das crianças e dos adolescentes, fato que foi constatado também por este estudo, quando visto que todos os estudantes utilizavam redes sociais. É importante ressaltar que os adolescentes se encontram em um momento no qual buscam a formação de sua identidade, além de estarem em processo de formação de vários aspectos da sua personalidade (Neves et al., 2015).

Quase a totalidade dos pesquisados referiu ser o celular o equipamento que mais utilizava para acessar a rede social. Achados similares foram relatados por Ferreira et al. (2017), que ao avaliarem 727 adolescentes verificaram que também utilizavam o celular com mais frequência para acesso à internet. Segundo Eisenstein e Estefenon (2011), os adolescentes de hoje fotografam tudo com seus sofisticados celulares, onde têm acesso a todas as informações. São ágeis, curiosos, informados e dominam a tecnologia.

$\mathrm{O}$ acesso às redes sociais é facilmente realizado através de telefones celulares, o que viabiliza o uso a qualquer momento, inclusive na hora de dormir. Nesta pesquisa verificou-se que os adolescentes que acessavam as redes sociais após as 22:00 horas apresentaram má qualidade do sono. Esses resultados corroboram com algumas pesquisas (Li, Kawachi, Buxton, Haneuse, \& Onnela, 2019; Fobian, Avis, \& Schwebel, 2016) que verificaram que a utilização de mídias sociais a noite repercutia negativamente no sono dos adolescentes, visto que altera a arquitetura do sono, comprometendo a sua eficiência e, consequentemente, a sua qualidade. Lemola, Perkinson-Gloor, Brand, Dewald-Kaufmann, e Grob (2015) também referiram que o uso de mídia eletrônica à noite está associado a distúrbios do sono, que também podem contribuir com o desenvolvimento de sintomas de depressão. Esses achados destacam a importância de evitar a utilização de uso de mídias eletrônicas à noite, a fim de obter uma melhor qualidade de sono, prevenindo dessa forma os efeitos adversos à saúde do adolescente.

Os estudantes que assistiam TV após as 10 horas da noite também apresentaram maior prevalência de má qualidade do sono. Dados semelhantes foram observados por Silva et al. (2017), em sua pesquisa com estudantes de 14 a 19 anos. Os autores verificaram que até oito horas de sono por dia e assistir TV por mais de duas horas/dia aumentam as chances de percepção negativa do sono. No estudo de Mesquita e Reimão (2007) observou-se a associação entre o uso de computadores durante a noite e a má qualidade do sono. Já Chinoy, Duffy e Czeisler (2018), perceberam a associação negativa da utilização e tablets à noite, repercutindo negativamente na qualidade do sono dos adolescentes.

Apesar de não ter sido observada associação entre a má qualidade do sono e o uso de jogos eletrônicos, foi observado que $70 \%$ dos pesquisados jogavam e desses, quase $40 \%$ jogavam todos os dias. A adolescência é caracterizada pela imaturidade dos sistemas cerebrais monoaminérgicos cortical frontal e subcortical, que faz com que a impulsividade seja um traço comportamental transitório típico dessa etapa da vida (van den Eijnden, Spijkerman, Vermulst, van Rooij, \& Engels, 2010). Esse dado pode explicar o fato de os adolescentes possuírem menos habilidades em controlar o entusiasmo por algo que lhes desperta interesse, estando mais vulneráveis ao uso patológico da internet (Ha et al., 2007; Terroso \& de Lima Argimon, 2016).

É importante destacar que as repercussões negativas sobre sono, devido ao uso das mídias digitais, vistas na presente 
pesquisa, acontecem também devido a emissão de luz azul dos celulares, tablets e notebooks, visto que a luminosidade suprime a produção da melatonina que regula o ritmo circadiano (Jniene et al., 2019; Calvo-Sanz e Tapia-Ayuga (2020). Por isso, contribuem para alterar a arquitetura do sono, desregulando o ritmo circadiano e trazendo as consequências de um sono de má qualidade, como a sonolência diurna excessiva, mau humor, ansiedade, depressão, sedentarismo, alimentação não saudável, obesidade, além de aumento de dores musculoesqueléticas, bruxismo e problemas oftalmológicos (Anjos et al., 2020; Perensin, Tieri, Marques, Godinho, \& Carpanez, 2020; Del Ciampo, Louro, Del Ciampo, \& Ferraz, 2017; Guedes, Desiderá \& Gonçalves, 2018; Felden et al., 2016).

Nossos achados trazem informações importantes do ponto de vista educacional e de saúde pública, mostrando que a baixa qualidade do sono em adolescentes pode estar relacionada ao uso excessivo de telas digitais. Ressalta-se que a associação entre qualidade do sono e uso de telas digitais é mediada pelo tempo de uso da internet. Assim, programas educacionais devem orientar os adolescentes para a redução do tempo de uso da internet visando a melhora da qualidade do sono. Faz-se necessário uma conscientização sobre os efeitos adversos à saúde causado pelo uso noturno e excessivo desses dispositivos pelos adolescentes.

\section{Conclusão}

Observou-se uma alta prevalência de má qualidade do sono e mais da metade dos alunos referiu falta de ânimo para executar as atividades diárias. $\mathrm{O}$ uso noturno de telas digitais e um tempo de uso $\geq 4$ horas/dia foram associados a uma má qualidade do sono.

Sugere- se que outros estudos sejam realizados com uma maior amostra de alunos, de diferentes municípios pernambucanos e com estudantes da rede privada de ensino para investigar se há diferença comportamental entre eles e os estudantes da rede pública.

\section{Referências}

Amra, B., Shahsavari, A., Shayan-Moghadam, R., Mirheli, O., Moradi-Khaniabadi, B., Bazukar, M., Yadollahi-Farsani, A., \& Kelishadi, R. (2017). The association of sleep and late-night cell phone use among adolescents. Jornal de pediatria, 93(6), 560-567.

Barbosa, F. D. O., Macedo, P. C. M., \& Silveira, R. M. C. D. (2011). Depressão e o suícido. Revista da SBPH, 14(1), $233-243$.

Barbosa, S. M. M. L., Batista, R. F. L., Rodrigues, L. D. S., Bragança, M. L. B. M., Oliveira, B. R. D., Simões, V. M. F., \& Silva, A. A. M. D. (2020). Prevalência de sonolência diurna excessiva e fatores associados em adolescentes da coorte RPS, em São Luís (MA). Revista Brasileira de Epidemiologia, 23, e200071.

Bertolazi, A. N., Fagondes, S. C., Hoff, L. S., Pedro, V. D., Menna Barreto, S. S., \& Johns, M. W. (2009). Portuguese-language version of the Epworth sleepiness scale: validation for use in Brazil. Jornal Brasileiro de Pneumologia, 35(9), 877-883.

Calvo-Sanz, J. A., \& Tapia-Ayuga, C. E. (2020). Blue light emission spectra of popular mobile devices: The extent of user protection against melatonin suppression by built-in screen technology and light filtering software systems. Chronobiology International, 37(7), 1016-1022.

Chinoy, E. D., Duffy, J. F., \& Czeisler, C. A. (2018). Unrestricted evening use of light-emitting tablet computers delays self-selected bedtime and disrupts circadian timing and alertness. Physiological reports, 6(10).

Dos Anjos, R. S., Cabral, L. F. S., Aguiar, L. D., Monteiro, V. R., de Menezes, V. A., Colares, V., da Franca, C.,\& de Godoy, F. (2020). Associação entre bruxismo e tempo de tela digital em adolescentes-Uma revisão integrativa. Brazilian Journal of Development, 6(7), 45896-45912.

Del Ciampo, L. A., Louro, A. L., Del Ciampo, I. R., \& Ferraz, I. S. (2017). Características de hábitos de sono entre adolescentes da cidade de Ribeirão Preto (SP). Revista Brasileira de Crescimento e Desenvolvimento Humano, 27(3).

Eisenstein, E., \& Estefenon, S. B. (2011). Geração digital: riscos das novas tecnologias para crianças e adolescentes. Revista Hospital Universitário Pedro Ernesto UERJ, 10, 42-53. http://bjhbs.hupe.uerj.br/WebRoot/pdf/105_pt.pdf

Felden, É. P. G., Filipin, D., Barbosa, D. G., Andrade, R. D., Meyer, C., Beltrame, T. S., \& Pelegrini, A. (2016). Adolescentes com sonolência diurna excessiva passam mais tempo em comportamento sedentário. Revista Brasileira de Medicina do Esporte, 22(3), 186-190.

Ferreira, C., Ferreira, H., Vieira, M. J., Costeira, M., Branco, L., Dias, Â., \& Macedo, L. (2017). Epidemiologia do Uso de Internet numa População Adolescente e Sua Relação com Hábitos de Sono. Acta médica portuguesa, 30. 
Fobian, A. D., Avis, K., \& Schwebel, D. C. (2016). The impact of media use on adolescent sleep efficiency. Journal of developmental and behavioral pediatrics: JDBP, 37(1), 9 .

Gajardo, Y. Z., Ramos, J. N., Muraro, A. P., Moreira, N. F., Ferreira, M. G., \& Rodrigues, P. R. M. (2021). Problemas com o sono e fatores associados na população brasileira: Pesquisa Nacional de Saúde, 2013. Ciência \& Saúde Coletiva, 26, 601-610.

Glanzmann, R., Moreira, L. F., de Almeida Marques, S., Silva, K. C., \& Soares, V. C. G. (2019). O uso da melatonina como indutor do sono-uma revisão bibliográfica. Revista uningá, 56(1), 157-167.

Guedes, D. P., Desiderá, R. A., \& Gonçalves, H. R. (2018). Prevalence of excessive screen time and correlates factors in Brazilian schoolchildren. Revista Brasileira de Atividade Física \& Saúde, 23, 1-10.

Ha, J. H., Kim, S. Y., Bae, S. C., Bae, S., Kim, H., Sim, M., Lyoo, I. K \& Cho, S. C. (2007). Depression and Internet addiction in adolescents. Psychopathology, 40(6), 424-430.

Instituto Brasileiro de Geografia e Estatística. (2010). Censo Demográfico https://www.ibge.gov.br/

Jniene, A., Errguig, L., El Hangouche, A. J., Rkain, H., Aboudrar, S., El Ftouh, M., \& Dakka, T. (2019). Perception of Sleep Disturbances due to Bedtime Use of Blue Light-Emitting Devices and Its Impact on Habits and Sleep Quality among Young Medical Students. BioMed research international, 2019.

Kosticova, M., Husarova, D., \& Dankulincova, Z. (2020). Difficulties in getting to sleep and their association with emotional and behavioural problems in adolescents: does the sleeping duration influence this association?. International journal of environmental research and public health, $17(5), 1691$.

Lemola, S., Perkinson-Gloor, N., Brand, S., Dewald-Kaufmann, J. F., \& Grob, A. (2015). Adolescents' electronic media use at night, sleep disturbance, and depressive symptoms in the smartphone age. Journal of youth and adolescence, 44(2), 405-418.

Li, X., Kawachi, I., Buxton, O. M., Haneuse, S., \& Onnela, J. P. (2019). Social network analysis of group position, popularity, and sleep behaviors among US adolescents. Social Science \& Medicine, 232, 417-426.

Lin, P. H., Lee, Y. C., Chen, K. L., Hsieh, P. L., Yang, S. Y., \& Lin, Y. L. (2019). The relationship between sleep quality and internet addiction among female college students. Frontiers in neuroscience, 13, 599.

Lira, D., \& Custodio, N. (2018). Los trastornos del sueño y su compleja relación con las funciones cognitivas. Revista de Neuro-Psiquiatría, 81(1), 20-28.

Lobo, D. C., Gigante, A. D., Navarro, C. M., \& Raso, V. (2017). Dependência de Internet sintomas depressivos em estudantes universitários. Colloquium Vitae, 9, 52-53.

Mesquita, G., \& Reimão, R. (2007). Nightly use of computer by adolescents: its effect on quality of sleep. Arquivos de neuro-psiquiatria, 65(2B), 428-432.

Munezawa, T., Kaneita, Y., Osaki, Y., Kanda, H., Minowa, M., Suzuki, K., Higuchi,S., Mori, J., Yamamoto, R., \& Ohida, T. (2011). The association between use of mobile phones after lights out and sleep disturbances among Japanese adolescents: a nationwide cross-sectional survey. Sleep, 34(8), 1013-1020.

Neves, K., Fosse, L., Torres, T., \& Napolitano, M. (2015). Da infância à adolescência: o uso indiscriminado das redes sociais. Rev. Ambiente Acadêmico, Cachoeiro de Itapemirim, 1(2), 119-139. de revista-ambiente-acadêmico-edicao-2-artigo-7.pdf (multivix.edu.br)

Otsuka, Y., Kaneita, Y., Itani, O., Jike, M., Osaki, Y., Higuchi, S., Kanda, H., Kinjo, A., Kuwabara, Y., \& Yoshimoto, H. (2020). The relationship between subjective happiness and sleep problems in Japanese adolescents. Sleep medicine, 69, 120-126.

Paruthi, S., Brooks, L. J., D'Ambrosio, C., Hall, W. A., Kotagal, S., Lloyd, R. M., \& Wise, M. S. (2016). Recommended amount of sleep for pediatric populations: a consensus statement of the American Academy of Sleep Medicine. Journal of clinical sleep medicine, 12(6), 785-786.

Passos, M. H., Silva, H. A., Pitangui, A. C., Oliveira, V., Lima, A. S., \& Araújo, R. C. (2017). Confiabilidade e validade da versão brasileira do Índice de Qualidade do Sono De Pittsburgh em adolescentes. Jornal de Pediatria, 93(2), 200-206.

Pereira, M. G. (1995). Epidemiologia: teoria e prática. Guanabara Koogan.

Perensin, F. S., Tieri, C. D. C. A., Marques, J. M., Godinho, C. L., \& Carpanez, L. R. (2020). Impacto do tempo de tela no de senvolvimento e qualidade de vida do adolescente. Unilus Ensino e Pesquisa, 17(48), 388-398.

Polos, P. G., Bhat, S., Gupta, D., O'Malley, R. J., DeBari, V. A., Upadhyay, H., Chaudhry, S., Nimma,A., Pinto-Zipp,G., \& Chokroverty, S. (2015). The impact of Sleep Time-Related Information and Communication Technology (STRICT) on sleep patterns and daytime functioning in American adolescents. Journal of adolescence, 44, 232-244.

Queiroz, L. B., Lourenço, B., Silva, L. E. V., Lourenço, D. M. R., \& Silva, C. A. (2018). Dor musculoesquelética e síndromes musculoesqueléticas em adolescentes relacionadas a dispositivos eletrônicos. Jornal de Pediatria, 94(6), 673-679.

Santos, E. D. S. G. D., \& Souza, O. F. D. (2021). Evidence of the association between sleep duration and blood pressure in adolescents: a systematic review. Revista Paulista de Pediatria, 39, 1-9.

Schmidek, H. C. M. V., Gomes, J. C., Santos, P. L. D., Carvalho, A. M. P. D., Pedrão, L. J., \& Corradi-Webster, C. M. (2018). Dependência de internet e transtorno de déficit de atenção com hiperatividade (TDAH): revisão integrativa. Jornal Brasileiro de Psiquiatria, 67(2), 126-134.

Silva, A. O. D., Oliveira, L. M. F. T. D., Santos, M. A. M. D., \& Tassitano, R. M. (2017). Tempo de tela, percepção da qualidade de sono e episódios de parassonia em adolescentes. Revista Brasileira de Medicina do Esporte, 23(5), 375-379.

Terroso, L. B., \& de Lima Argimon, I. I. (2016). Dependência de internet e habilidades sociais em adolescentes. Estudos e pesquisas em psicologia, 16(1), 200-219. 
Research, Society and Development, v. 10, n. 7, e43810716714, 2021

(CC BY 4.0) | ISSN 2525-3409 | DOI: http://dx.doi.org/10.33448/rsd-v10i7.16714

Van Den Eijnden, R. J., Spijkerman, R., Vermulst, A. A., van Rooij, T. J., \& Engels, R. C. (2010). Compulsive Internet use among adolescents: Bidirectional parent-child relationships. Journal of abnormal child psychology, 38(1), 77-89.

Woods, H. C., \& Scott, H. (2016). \# Sleepyteens: Social media use in adolescence is associated with poor sleep quality, anxiety, depression and low selfesteem. Journal of adolescence, 51, 41-49.

Zhang, Y. Y., Chen, J. J., Ye, H., \& Volantin, L. (2020). Psychological effects of cognitive behavioral therapy on internet addiction in adolescents: A systematic review protocol. Medicine, 99(4). 